\title{
Erratum to: Characterization of water reservoirs affected by acid mine drainage: geochemical, mineralogical, and biological (diatoms) properties of the water
}

\author{
T. Valente ${ }^{1,2} \cdot$ M. J. Rivera ${ }^{1,2} \cdot$ S. F. P. Almeida ${ }^{3}$ - C. Delgado ${ }^{3} \cdot$ P. Gomes $^{1,2}$. $^{2}$ \\ J. A. Grande ${ }^{2}$ - M. L. de la Torre ${ }^{2}$ - M. Santisteban ${ }^{1,2}$
}

Published online: 25 July 2015

(C) Springer-Verlag Berlin Heidelberg 2015

Erratum to: Environ Sci Pollut Res

DOI 10.1007/s11356-015-4776-0

The following errors have been detected in figures 4 and 5 and table 3. They represent issues of style but they have no influence on the results.

In table 3 the list of identified taxa in the studied sites was replaced by:

Achnanthes lanceolata (Brébisson) Grunow var. lanceolata

Bacillaria paradoxa Gmelin

Brachysira neoexilis Lange-Bertalot

Cyclotella meneghiniana Kützing

Diploneis sp.

Diploneis separanda Lange-Bertalot

Discostella pseudostelligera (Hustedt) Houk \& Klee

Eunotia exigua (Brébisson ex Kützing) Rabenhorst

Gomphonema parvulum (Kützing) Kützing var. parvulum

Navicula gregaria Donkin

Navicula halophila (Grunow) Cleve

Navicula veneta Kützing

Navicula sp.

The online version of the original article can be found at http://dx.doi.org/ 10.1007/s11356-015-4776-0.

T. Valente

teresav@dct.uminho.pt

1 ICT, Institute of Earth Sciences, Pole of the University of Minho, Campus de Gualtar, 4710-057 Braga, Portugal

2 CIPIMS, Universidad de Huelva, Campus de la Rábida, Ctra. Palos de la Frontera s/n. 21819, Palos de la Frontera, Huelva, Spain

3 Departament of Biology and GEOBIOTEC, University of Aveiro, Campus de Santiago, 3810-193 Aveiro, Portugal
Nitzschia amphibia Grunow f. amphibia

Nitzschia palea (Kützing) W. Smith

Nitzschia cf. thermalis (Kützing) var. minor Hilse

Pinnularia acidophila Hoffman \& Krammer

Pinnularia aljustrelica Luis, Almeida \& Ector

Pinnularia acoricola Hustedt var. acoricola

Pinnularia subcapitata Gregory var. subcapitata

In Fig. 4. Dominant and sub-dominant diatom taxa in different $\mathrm{pH}$ ranges, the list of taxa was replaced by:

Discostella pseudostelligera (Hustedt) Houk \& Klee

Achnanthes lanceolata (Brébisson) Grunow var. lanceolata

Cyclotella meneghiniana Kützing

Bacillaria paradoxa Gmelin

Diploneis sp.

Gomphonema parvulum (Kützing) Kützing var. parvulum

Navicula gregaria Donkin

Navicula halophila (Grunow) Cleve

Navicula veneta Kützing

Navicula sp.

Nitzschia amphibia Grunow f. amphibia

Nitzschia cf. thermalis Kützing var. minor Hilse

Eunotia exigua (Brébisson ex Kützing) Rabenhorst

Diploneis separanda Lange-Bertalot

Brachysira neoexilis Lange-Bertalot

Pinnularia subcapitata Gregory var. subcapitata

Pinnularia aljustrelica Luis, Almeida \& Ector

Pinnularia acidophila Hofmann \& Krammer

Pinnularia acoricola Hustedt var. acoricola

Fig. 5 was replaced by a new figure with the following caption:

Fig. 5 Images of diatoms obtained under the light microscope (Zeiss Axioplan 2 Imaging). Scale Bar $10 \mu \mathrm{m}$.

1-3 Pinnularia aljustrelica Luis, Almeida \& Ector

4-5 Pinnularia acidophila Hofmann \& Krammer 
6 Pinnularia aff. acoricola Hustedt var. acoricola 7-8 Nitzschia palea (Kützing) W. Smith

9 Nitzschia cf. thermalis Kützing var. minor Hilse 10-11 Pinnularia aff. subcapitata Gregory var. subcapitata 12 Cyclotella meneghiniana Kützing
13 Discostella pseudostelligera (Hustedt) Houk \& Klee 14 Navicula veneta Kützing

15 Navicula gregaria Donkin

16 Nitzschia amphibia Grunow f. amphibia

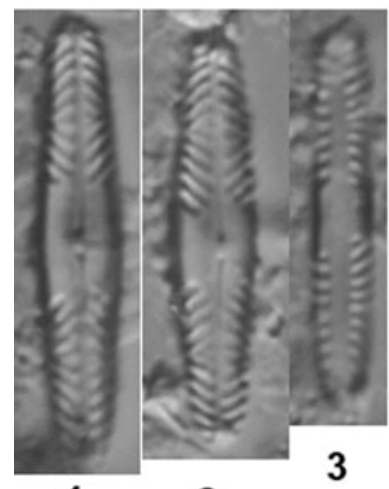

1

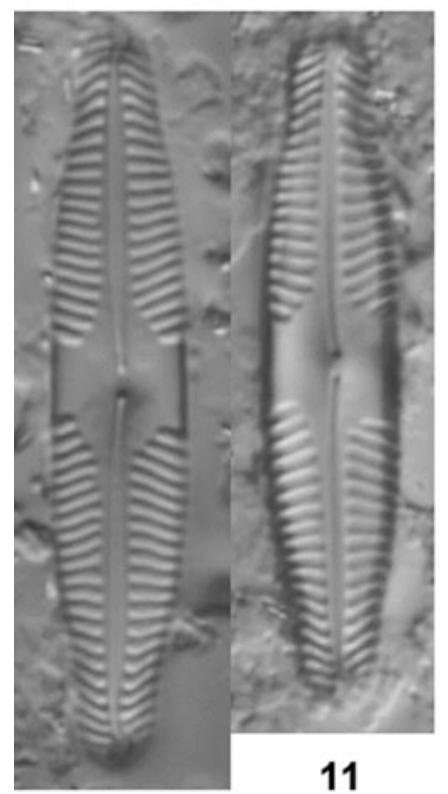

10
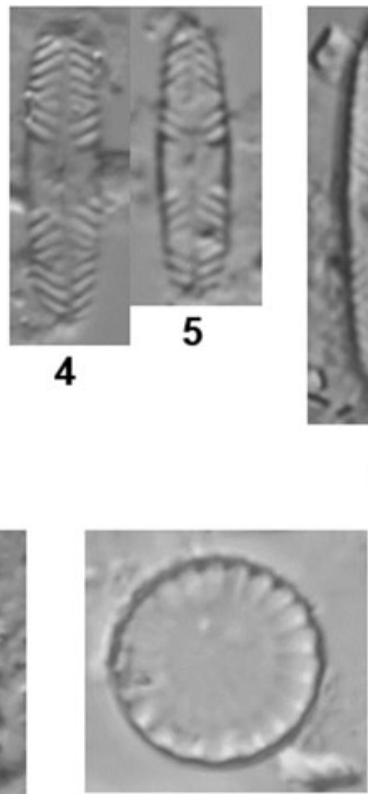

12

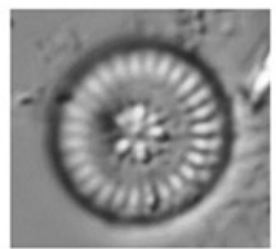

13

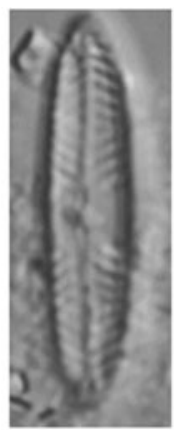

6
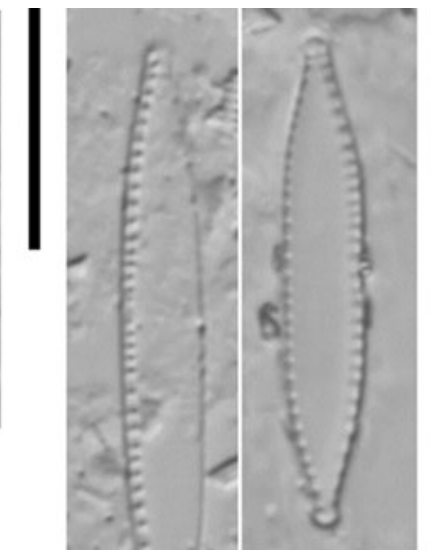

8

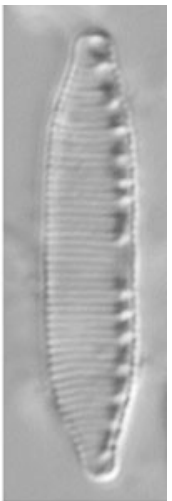

9

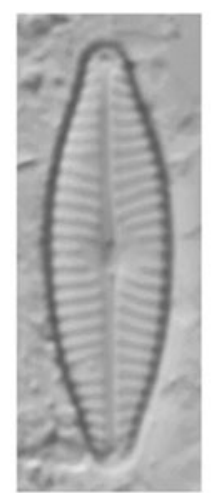

14
16

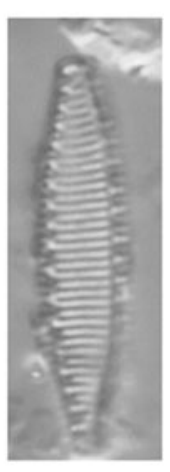

15

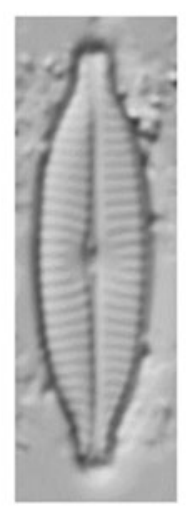

7

6

Fig. 5 